\title{
Field Dependent Avalanche Ionization Rates in Dielectrics
}

\author{
P. P. Rajeev, ${ }^{1}$ M. Gertsvolf, ${ }^{1,2}$ P. B. Corkum, ${ }^{1,2, *}$ and D. M. Rayner ${ }^{1, \dagger}$ \\ ${ }^{1}$ National Research Council of Canada, Ottawa, Canada, K1A OR6 \\ ${ }^{2}$ University of Ottawa, Ottawa, Canada, K1N 6N5 \\ (Received 28 April 2008; published 24 February 2009)
}

From the pulse length dependence of the absorption of intense ultrashort laser pulses focused inside fused silica, we reveal the role field-assisted collisional ionization plays in the multiphoton ionization process. This constitutes a cold avalanche ionization mechanism that persists at pulse lengths considered too short for a traditional avalanche.

DOI: 10.1103/PhysRevLett.102.083001

PACS numbers: $33.80 . \mathrm{Rv}, 42.65 .-\mathrm{k}$

Collision- and photon-assisted electron tunneling are important, related processes in intense field physics. In the nonresonant multiphoton ionization of atoms, recollision electrons can stimulate nonsequential double ionization-the single atom analogue to avalanche ionization [1]. It is now recognized that ionization can also occur for a low energy collision where the intense laser field assists the collision [2]. In other words, the recollision creates a double excited state that has a significantly lowered tunneling barrier and consequently ionizes readily. In solidstate device physics, photon-assisted tunneling is well known. Here it is adsorption of a photon rather than a collision that contributes the energy, but the effect in both cases is lowering of the tunneling barrier. Photonassisted tunneling is also applied in scanning tunneling microscopy [3]. We show that collision-assisted tunneling (or field-assisted collisional ionization) also plays a role when intense laser pulses interact with dielectric materials.

Collision-driven avalanche ionization is accepted to dominate laser dielectric interactions at pulse lengths $>100 \mathrm{fs}[4,5]$. On this time scale, there is sufficient time for efficient inverse bremsstrahlung heating to produce electrons with kinetic energy above the band gap of the material. With relatively long pulses (10 ps to $1 \mathrm{~ns})$, thermal electrons seed the avalanche. As the pulse is shortened and made more intense, multiphoton ionization begins to seed the avalanche, making the interaction more deterministic [5,6]. Some estimates, based on the Drude model, imply that avalanche ionization is unimportant under $100 \mathrm{fs}$, electron heating being limited by the electronphonon collision rate [7]. In contradiction to these models, there are experimental reports of avalanche ionization operating for pulses as short as $10 \mathrm{fs}$ in fused silica [8].

The majority of previous studies of avalanches are based on surface damage threshold measurements. We measure the nonlinear absorption of the ionizing pulse. By placing the focus inside the dielectric, we are able to study the interaction over a range of pulse durations while remaining well below the damage threshold, avoiding subjective measurements of the damage threshold. The precision and range of our measurements allow us to distinguish a field-assisted collisional (cold) avalanche from a traditional avalanche and show how the latter becomes dominant for longer pulses. Field-assisted collisional ionization has been proposed theoretically as a mechanism that can drive avalanche ionization even in a few-cycle pulse [9].

We use $800 \mathrm{~nm}$ laser pulses from a regenerative amplifier operated at $40 \mathrm{~Hz}$. The pulses are focused $150 \mu \mathrm{m}$ inside the fused silica sample (Suprasil I, Boston Piezo-
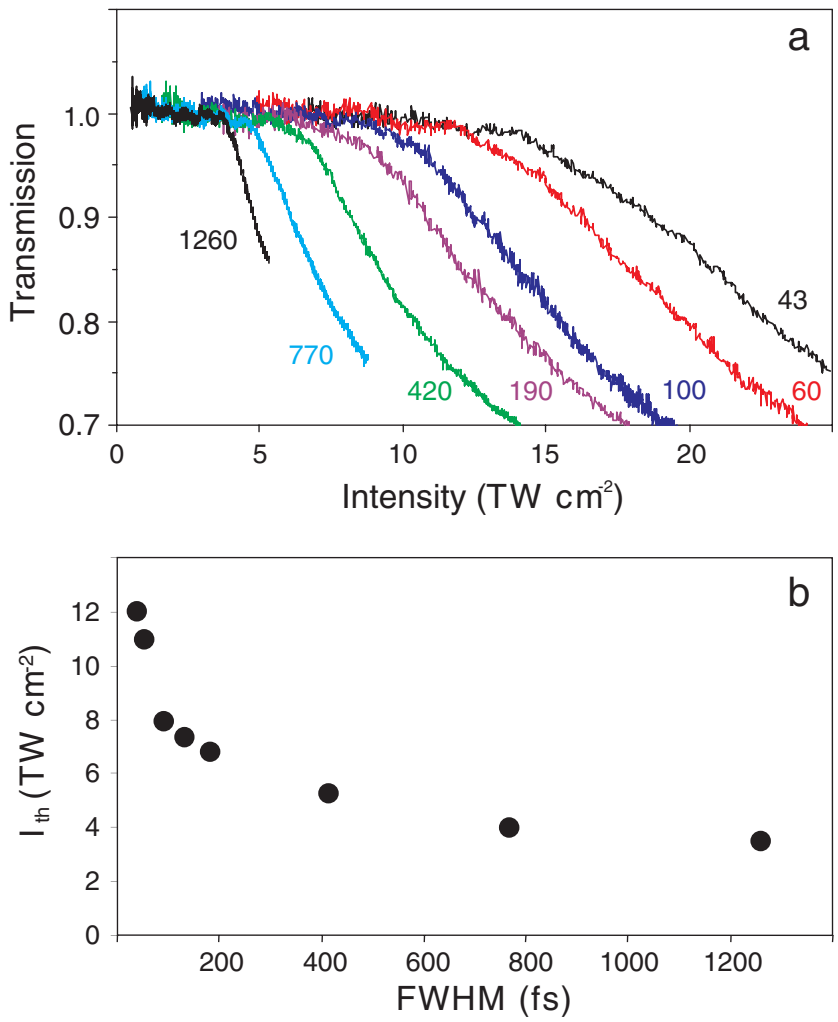

FIG. 1 (color online). (a) Pulse width dependence of the transmission of femtosecond laser pulses through a tight focus in fused silica as a function of pulse peak intensity that would have been reached if the focus were in vacuum. The curves are labeled by their FWHM in femtoseconds. (b) Threshold intensities $I_{\text {th }}$ obtained from such transmission curves, as a function of pulse width. 
Optics, Inc.) using a $0.25 \mathrm{NA}$ microscope objective. The pulse duration at the focus $\tau$ is varied between $\sim 40$ fs and $\sim 1$ ps using a linear chirp controlled by the pulse compressor. Our results are not dependent on the chirp sign. The pulse duration was measured using a noncollinear autocorrelator assuming a Gaussian pulse shape. A combination of a half-wave plate and a polarizer controls the intensity of incident light. We use a split beam configuration that employs integrating spheres to monitor both the incident and the transmitted light. The sample is moved continuously, orthogonal to the laser beam, such that each shot is incident on fresh material to avoid memory effects in transmission [10]. Consistent with the high homogeneity of Suprasil I, we have not found any inconsistencies that can be attributed to preexisting defects in the dielectric. The transmission is measured on a shot-to-shot basis, acquiring every laser pulse using a computer-controlled data acquisition system.

Figure 1(a) shows the transmission through fused silica as a function of the incident pulse intensity (if the focus were in vacuum) for pulses of duration between 43 and 1260 fs full width at half maximum (FWHM). The transmitted signal remains constant (no absorption) for low laser intensities. When the intensity exceeds the level required for material breakdown, strong nonlinear absorption occurs that leads to a sharp drop in the transmission. The intensity that corresponds to the inflection in the curve is the threshold intensity $I_{\text {th }}$. For a systematic analysis of $I_{\mathrm{th}}(\tau)$, we extract its value using the analytical model presented in Ref. [11]. The results are summarized in Fig. 1(b).

Before proceeding, it is important to note that our results are not affected by propagation effects such as selffocusing and self-phase modulation because the pulse power $P$ is kept below the critical power for self-focusing $\left(P_{C} \approx 1.7 \mathrm{MW}\right.$ in fused silica, or $75 \mathrm{~nJ}$ for a $45 \mathrm{fs}$ pulse). In addition, nonlinear absorption lowers the peak power long before the focal region. This is confirmed by the absence of self-phase modulation in the transmitted spectrum [11].

If multiphoton ionization, an intensity-dependent process, were the only absorption mechanism, $I_{\text {th }}$ should remain nearly constant with $\tau$. However, we find that $I_{\text {th }}$ decreases 3 times with a $\sim 20$ times increase in $\tau$. The dependence of $I_{\mathrm{th}}$ on $\tau$ is the signature of avalanche ionization just as the $\tau$ dependence of the damage threshold on surfaces is $[4,8]$.

Nonlinear absorption in dielectrics is related to the production and density of carriers. Generally, the carrier generation rate is given by

$$
\frac{d n}{d t}=W(I)+\alpha I n
$$

where $\alpha$ is the avalanche coefficient and $W(I)$ is the multiphoton ionization rate [4]. Here we neglect electron trapping. We can do this, even though our pulse lengths exceed the trapping time in fused silica, because trapping is countered by immediate reionization of the trapped states resulting in no net change in $n$. Reionization is rapid because the lowest trap state still lies $3.5 \mathrm{eV}$ above the ground state [12].

We fit the experimental transmission curves by numerical analysis of the pulse propagation through the focus, taking nonlinear absorption and carrier generation into account through Eq. (1) $[11,13]$. The model assumes that beam propagation is not significantly altered by selffocusing (as we have discussed) or plasma refractive effects. The latter assumption is valid only for relatively low plasma densities. Therefore, we expect the model to work best near the threshold. It will deviate at high absorption levels when the plasma density becomes large. We model $W(I)$ as $\sigma_{k} I^{k}$, where $k$ is the multiphoton order and $\sigma_{k}$ is the $k$ th order cross section. Setting $k$ to correspond to the band gap, we adjust $\sigma_{k}$ and $\alpha$ to fit the model to the experiment. The transmission curve for a 40 fs pulse has been described well by this approach with $k=6, \sigma_{6}=$ $4 \times 10^{13}\left(\mathrm{TW} \mathrm{cm}^{-2}\right)^{-6} \mathrm{~cm}^{-3} \mathrm{ps}^{-1}$, and $\alpha=4 \mathrm{~J}^{-1} \mathrm{~cm}^{2}$ in fresh fused silica [10], similar to that predicted by the Keldysh theory for a band gap of $9 \mathrm{eV}$ [14].

Using the above parameters for $W(I)$ and $\alpha$, Fig. 2(a) compares fits (long-dashed, red line) made to transmission
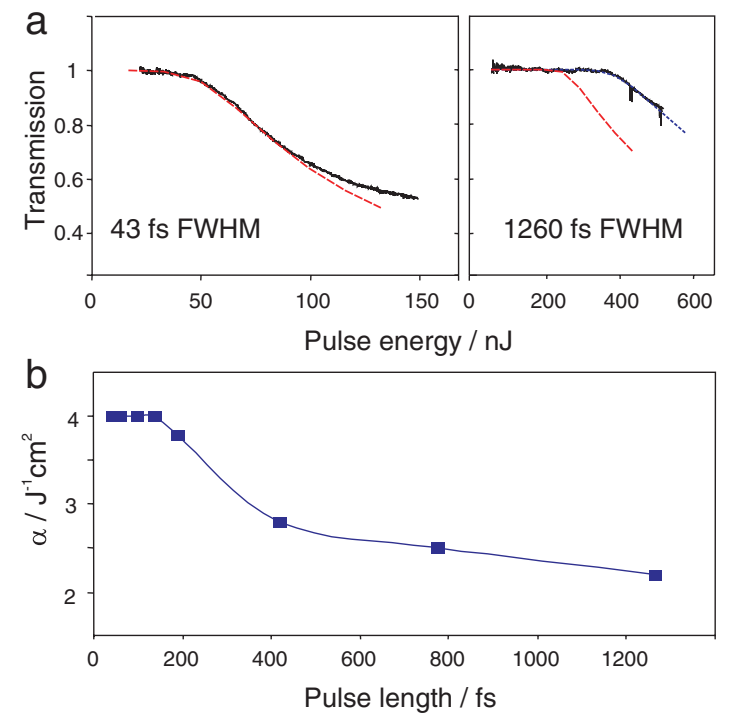

FIG. 2 (color online). (a) Numerical fits to the transmission vs pulse energy curves for pulses of 43 and 1260 fs FWHM including a traditional avalanche through Eq. (1). The solid, noisy curves are the experimental results. The red, long-dashed line in both panels is obtained with $\alpha=4 \mathrm{~J}^{-1} \mathrm{~cm}^{2}$. The blue, short-dashed line in the left panel is obtained with $\alpha=$ $2.2 \mathrm{~J}^{-1} \mathrm{~cm}^{2}$. In both cases the multiphoton rate $W(I)$ is taken as $\sigma_{6} I^{6}$, with $\sigma_{6}=4 \times 10^{13}\left(\mathrm{TW} \mathrm{cm}^{-2}\right)^{-6} \mathrm{~cm}^{-3} \mathrm{ps}^{-1}$. The transmission curves cannot be modeled globally using a single value of $\alpha$. (b) The avalanche coefficient $\alpha$ derived as a function of pulse length by fitting the transmission curves, keeping $\sigma_{6}$ fixed. 
curves (solid, black line) collected using 43 and $1260 \mathrm{fs}$ pulses. The agreement with the experiment is good only for $\tau=43 \mathrm{fs}$. As discussed above, in all of our analysis we concentrate on absorption around $I_{\text {th }}$ where focusing remains unchanged with the interaction. For $1260 \mathrm{fs}$, using the same parameters underestimates the experimental threshold by a factor of almost 2. Also shown is the fit (short-dashed, blue line) to the $1260 \mathrm{fs}$ data, using the same value of $\sigma_{6}$ but smaller $\alpha=2.2 \mathrm{~J}^{-1} \mathrm{~cm}^{2}$. This fits the data well. A sensitivity analysis shows that $\sigma_{6}$ has to be changed by an unrealistic amount to have the same effect as the factor of $\sim 2$ change in $\alpha$.

We repeat the fitting procedure, keeping fixed $\sigma_{6}$ value and adjusting $\alpha$ for all curves shown in Fig. 1(a). Figure 2(b) shows the dependence of $\alpha$ on pulse length. The results demonstrate, perhaps unexpectedly, that the avalanche rate is higher for shorter pulses.

We propose that the increase in $\alpha$ with a decrease in pulse duration is the signature of a photon-assisted (or cold) avalanche. The higher the light intensity, the less energy an electron needs to cause field-assisted collisional ionization. Consequently, the process should become increasingly more efficient for shorter pulses. As a result, the apparent avalanche coefficient derived from experiment is expected to rise with the peak pulse intensity.

To include photon-assisted avalanche we modify the carrier generation rate [Eq. (1)]:

$$
\frac{d n}{d t}=W(I)+\alpha_{0} \operatorname{In}\left(1+\sum_{j=1}^{k-1} \beta_{j} I^{j}\right)
$$

where $\alpha_{0}$ is now the traditional avalanche coefficient. In the brackets we include the multiphoton contribution to collisional ionization with "colder" electrons $\beta_{j} I^{j}$. The colder the electrons, the higher the multiphoton order $j$.

During photon-assisted collisional ionization, the multiphoton contribution should have a dominant order of $j$ depending on the laser intensity. A unique feature of experiments performed inside the material is that the maxi- mum intensity reached is clamped at the level of $I_{\text {th }}$ by nonlinear absorption. In the case of fused silica, $I_{\text {th }} \leq$ $12 \mathrm{TW} \mathrm{cm} \mathrm{cm}^{-2}$ [Fig. 2(b)]. At this intensity the photon flux is $\sim 1$ photon per $\AA^{-2}$ per 300 as, i.e., one photon in the expected lifetime of the electron-atom collision complex. Higher orders of $j$ are much less likely in this intensity range, making the $j=1$ term in Eq. (2) dominant under our conditions.

Figure 3 shows the result of a global fit to all of the transmission curves using single values of $\alpha_{0}$ and $\beta_{1}$, independent of $\tau$. The fits were obtained with $\alpha_{0}=$ $1.7 \mathrm{~J}^{-1} \mathrm{~cm}^{2}$ and $\beta_{1}=\beta=0.1 \mathrm{TW}^{-1} \mathrm{~cm}^{2}$. Again we approximate $W(I)=\sigma_{k} I^{k}$ with $k=6$ and $\sigma_{6}=4 \times$ $10^{13}\left(\mathrm{TW} \mathrm{cm}^{-2}\right)^{-6} \mathrm{~cm}^{-3} \mathrm{ps}^{-1}$. Considering the global nature of the fit and the uncertainty in the pulse width measurement $(<15 \%)$, the agreement is good. By including a single, low order term to describe a cold avalanche, we successfully model nonlinear absorption in fused silica for pulses from 43 to 1260 fs. Our last task is to modify the flux-doubling model [4] to account for photon-assisted collisional ionization. This will allow us to naturally extend our description of a photon-assisted avalanche to the higher intensities that characterize lasersurface interactions.

Returning to Eq. (2), the product $\alpha_{0} I$ represents the electron heating rate divided by the threshold for collisional ionization. Under the flux-doubling approximation, $\alpha_{0}$ is given by

$$
\alpha_{0}=\frac{2 p}{\varepsilon_{0} c \int_{0}^{U_{\mathrm{th}}} 1 / \sigma(\epsilon) d \epsilon},
$$

where $p$ takes a value between 0.5 and $1, U_{\text {th }}$ is the electron energy threshold for collisional ionization, and $\sigma(\epsilon)$ is the conductivity per electron at electron energy $\epsilon$, given under the Drude formalism by

$$
\sigma=\frac{e^{2}}{\omega m^{*}} \frac{\tau_{c}(\epsilon) \omega}{\left[1+\tau_{c}(\epsilon)^{2} \omega^{2}\right]} .
$$

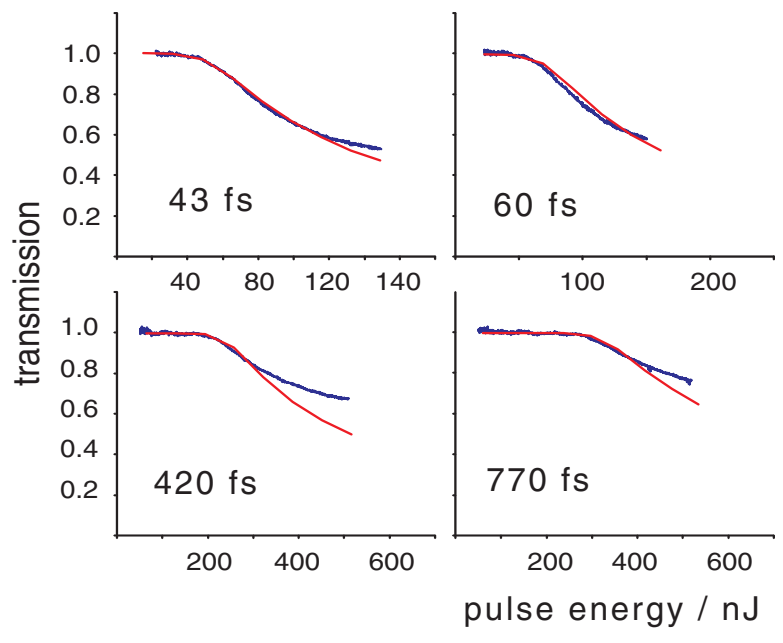

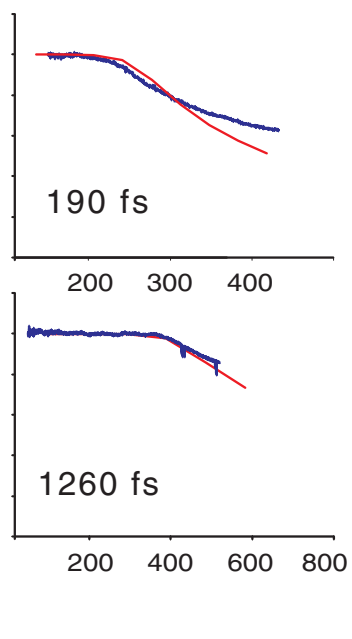

FIG. 3 (color online). Transmission vs pulse energy curves for pulses in the range 43-1260 fs FWHM. The solid, noisy curves are the experimental results. The red, long-dashed lines are fits obtained globally by including a cold avalanche through the carrier generation rate given by Eq. (2), with $\alpha_{0}=1.7 \mathrm{~J}^{-1} \mathrm{~cm}^{2}$, $\beta=0.1 \mathrm{TW}^{-1} \mathrm{~cm}^{2}$, and $j=1$. The multiphoton rate $W(I)$ is taken as $\sigma_{6} I^{6}$, with $\sigma_{6}=4 \times 10^{13}\left(\mathrm{TW} \mathrm{cm}^{-2}\right)^{-6} \mathrm{~cm}^{-3} \mathrm{ps}^{-1}$. 


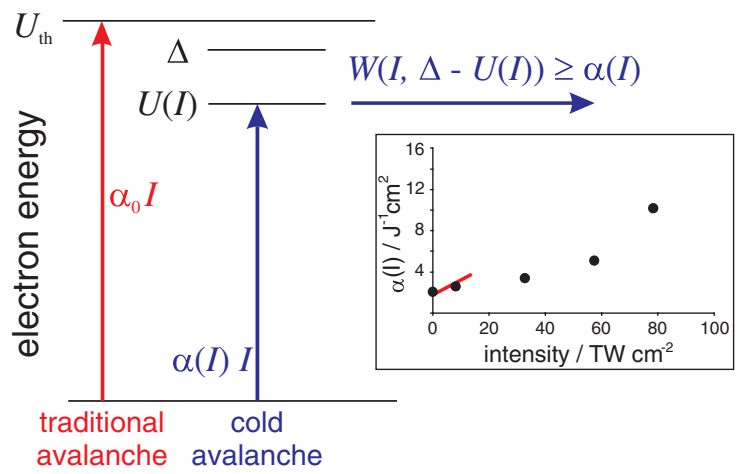

FIG. 4 (color online). Schematic rate description of traditional and cold avalanche ionization. $U_{\text {th }}$ is the collisional ionization threshold. In the absence of an external field, the avalanche rate $\alpha_{0} I$ is determined by the heating rate to this level. In the presence of an external field, the effective threshold $U(I)$ is determined as the energy at which the multiphoton ionization rate $W(I, \Delta-U(I))$ exceeds the heating rate to that energy level; i.e., the ionization probability approaches $1 . \Delta$ is the band gap. Inset: Dependence of the avalanche coefficient $\alpha$ on intensity: (black dots) estimated using the flux-doubling model; (red line) derived from the fit parameters in Fig. 3.

Here $e$ is the electron charge, $\omega$ is the laser frequency, $m^{*}$ is the reduced mass of the electron, and $1 / \tau_{c}(\epsilon)$ is the electron energy, $\epsilon$ dependent electron-phonon momentum collision rate. For $\tau_{c}(\epsilon)$ independent of $\epsilon$, and with $p=1, \alpha$ becomes simply $2 \sigma / \varepsilon_{0} c U_{\text {th }}$.

We propose that, in the flux-doubling context, cold avalanche lowers the effective collisional ionization threshold. The concept is shown schematically in Fig. 4. In this picture, a traditional avalanche is described by the pumping rate $\alpha_{0} I$ to the threshold $U_{\text {th }}$ where collisional ionization is effectively instantaneous. In the presence of a strong laser pulse, this threshold is lowered due to field assistance. The extent depends on the field.

The threshold in the presence of the field $U(I)$ is the electron energy at which the heating rate is exceeded by the collision-assisted ionization rate $W(I, \Delta-U(I))$. This recognizes that the ionization rate $W$ is a function of the effective band gap $\Delta-U(I)$ as well as the intensity $I$. $W(I, \Delta-U(I))$ increases as the effective band gap decreases. We can determine the intensity dependence of the avalanche coefficient by replacing $U_{\text {th }}$ with $U(I)$ in Eq. (3). If we assume $\tau_{c}$ is independent of $\epsilon$, with $p=1$, the result is $\alpha(I)=2 \sigma / \varepsilon_{0} c U(I)$ or, in terms of $\alpha_{0}$,

$$
\alpha(I)=\frac{\alpha_{0} U_{\mathrm{th}}}{U(I)} .
$$

The inset in Fig. 4 shows the result for $\alpha(I)$ obtained by comparing multiphoton rates based on the Keldysh formalism with $\alpha(I) I$ from Eq. (5) (black dots). At low intensities there is reasonable agreement with what we found using the first-order term $\alpha(I)=\alpha_{0}(1+\beta I)$ (solid red line) of the perturbation expansion in Eq. (2) using the values of $\alpha_{0}$ and $\beta$ from Fig. 3. From a perturbative perspective, higher order terms will be needed at higher intensities (physically, there are more photons interacting during the lifetime of the collision complex). The curvature of $\alpha(I)$ vs $I$ is consistent with including higher orders of $j$ in Eq. (2). Both approaches agree with the intuitive conclusion-the order of the field assistance in the cold avalanche process must increase under higher intensity.

In conclusion, we have demonstrated a method of studying light interaction with dielectrics that has sufficient precision to confirm a field-assisted avalanche. This extends the concept of field-assisted collisional ionization all the way from single atoms to solids. In bulk dielectrics, the light intensity never becomes very large, so field-assisted tunneling increases the avalanche coefficient by only a factor of $\sim 2$. However, at the surface of dielectrics, the situation is different. High intensities can be reached because the pulse is not attenuated before the focus by nonlinear absorption. The order of assistance to the collision will be much higher in this case. Comprehensive models for laser machining and laser damage in dielectrics need to take the photon-assisted avalanche into account.

*Paul.Corkum@nrc.gc.ca

†David.Rayner@nrc.gc.ca

[1] P. B. Corkum, Phys. Rev. Lett. 71, 1994 (1993).

[2] V. R. Bhardwaj, S. A. Aseyev, M. Mehendale, G. L. Yudin, D. M. Villeneuve, D. M. Rayner, M. Y. Ivanov, and P. B. Corkum, Phys. Rev. Lett. 86, 3522 (2001).

[3] S. Grafström, J. Appl. Phys. 91, 1717 (2002).

[4] B. C. Stuart, M. D. Feit, S. Herman, A. M. Rubenchik, B. W. Shore, and M. D. Perry, Phys. Rev. B 53, 1749 (1996).

[5] A.-C. Tien, S. Backus, H. Kapteyn, M. Murnane, and G. Mourou, Phys. Rev. Lett. 82, 3883 (1999).

[6] B. C. Stuart, M. D. Feit, A. M. Rubenchik, B. W. Shore, and M. D. Perry, Phys. Rev. Lett. 74, 2248 (1995).

[7] F. Quéré, S. Guizard, and P. Martin, Europhys. Lett. 56, 138 (2001).

[8] M. Lenzner, J. Kruger, S. Sartania, Z. Cheng, C. Spielmann, L. G. Mourou, W. Kautek, and F. Krausz, Phys. Rev. Lett. 80, 4076 (1998).

[9] L. N. Gaier, M. Lein, M. I. Stockman, G. L. Yudin, P. B. Corkum, M. Y. Ivanov, and P. L. Knight, J. Mod. Opt. 52, 1019 (2005).

[10] P. P. Rajeev, M. Gertsvolf, E. Simova, C. Hnatovsky, R. S. Taylor, V. R. Bhardwaj, D. M. Rayner, and P. B. Corkum, Phys. Rev. Lett. 97, 253001 (2006).

[11] D. M. Rayner, A. Naumov, and P. B. Corkum, Opt. Express 13, 3208 (2005).

[12] K. Tanimura, T. Tanaka, and N. Itoh, Phys. Rev. Lett. 51, 423 (1983).

[13] L. Sudrie, A. Couairon, M. Franco, B. Lamouroux, B. Prade, S. Tzortzakis, and A. Mysyrowicz, Phys. Rev. Lett. 89, 186601 (2002).

[14] L. V. Keldysh, Sov. Phys. JETP 20, 1307 (1965). 